\title{
Interventional multispectral photoacoustic imaging with a clinical linear array ultrasound probe for guiding nerve blocks
}

\author{
Wenfeng Xia, ${ }^{1, *}$ Simeon J. West, ${ }^{2}$ Daniil I. Nikitichev, ${ }^{1}$ Sebastien Ourselin, ${ }^{1}$ Paul C. Beard, ${ }^{1}$ \\ and Adrien E. Desjardins ${ }^{1}$ \\ ${ }^{1}$ Department of Medical Physics and Biomedical Engineering, University College London, \\ Gower street, London WC1E 6BT, United Kingdom; \\ ${ }^{2}$ Main Theatres, Maple Bridge Link Corridor, Podium 3, University College Hospital, 235 \\ Euston Rd, London NW1 2BU, United Kingdom
}

\begin{abstract}
Accurate identification of tissue structures such as nerves and blood vessels is critically important for interventional procedures such as nerve blocks. Ultrasound imaging is widely used as a guidance modality to visualize anatomical structures in real-time. However, identification of nerves and small blood vessels can be very challenging, and accidental intra-neural or intra-vascular injections can result in significant complications. Multi-spectral photoacoustic imaging can provide high sensitivity and specificity for discriminating hemoglobin- and lipid-rich tissues. However, conventional surface-illumination-based photoacoustic systems suffer from limited sensitivity at large depths. In this study, for the first time, an interventional multispectral photoacoustic imaging (IMPA) system was used to image nerves in a swine model in vivo. Pulsed excitation light with wavelengths in the ranges of $750-900 \mathrm{~nm}$ and $1150-1300 \mathrm{~nm}$ was delivered inside the body through an optical fiber positioned within the cannula of an injection needle. Ultrasound waves were received at the tissue surface using a clinical linear array imaging probe. Co-registered B-mode ultrasound images were acquired using the same imaging probe. Nerve identification was performed using a combination of B-mode ultrasound imaging and electrical stimulation. Using a linear model, spectral-unmixing of the photoacoustic data was performed to provide image contrast for oxygenated and de-oxygenated hemoglobin, water and lipids. Good correspondence between a known nerve location and a lipid-rich region in the photoacoustic images was observed. The results indicate that IMPA is a promising modality for guiding nerve blocks and other interventional procedures. Challenges involved with clinical translation are discussed.
\end{abstract}

KEYWORDS: Photoacoustic imaging; Ultrasound imaging; Spectroscopy; Nerve blocks; Medical device guidance

\section{INTRODUCTION}

Accurate identification of critical tissue structures is of great importance for interventional procedures. With nerve blocks, these structures include nerves and blood vessels. Ultrasound (US) imaging is widely used for realtime guidance. However, US imaging can provide insufficient image contrast for soft tissue structures such as nerves and blood vessels, even when power Doppler is used. ${ }^{1,2}$ Accidental intra-neural or intra-vascular injections can result in significant complications. ${ }^{3}$

Several methods have been used to facilitate nerve identification in clinical practice. ${ }^{1}$ Traditionally paraesthesia was used, in which contact between the needle and the nerve was detected by the patient. Nerve stimulators are used in current clinical practice, however with this method it is still not possible to completely avoid complications and therefore alternative methods are highly desired.

Optical detection of nerves has attracted intensive research interest in recent years. These optical methods include optical reflectance spectroscopy, ${ }^{4-6}$ confocal imaging, ${ }^{7}$ coherent anti-Stokes Raman scattering microscopy, ${ }^{8}$ optical coherence tomography, ${ }^{9}$ and fluorescence imaging with exogeneous contrast agents. ${ }^{10}$ However, these imaging modalities lack sufficient imaging depth for clinical applications due to strong light absorption and scattering in biological tissues. Photoacoustic imaging is a solution to this problem. ${ }^{11}$ Conventional PA imaging

* Address all correspondence to: Wenfeng Xia, Department of Medical Physics and Biomedical Engineering, University College London, Malet Place Engineering Building, London WC1E 6BT, United Kingdom; Tel: +44 20 76790300; E-mail: wenfeng.xia@ucl.ac.uk.

Photons Plus Ultrasound: Imaging and Sensing 2016, edited by Alexander A. Oraevsky, Lihong V. Wang Proc. of SPIE Vol. 9708, 97080C - (c) 2016 SPIE · CCC code: 1605-7422/16/\$18 - doi: 10.1117/12.2209047 
systems deliver excitation light at the tissue surface. The sensitivity of imaging systems decreases exponentially with depth; imaging beyond a few centimeters in depth presents significant challenges. ${ }^{12}$ Interventional PA imaging systems address this issue by delivering light close to the region of interest and detecting US waves at the tissue surface. ${ }^{13-17}$ In the authors' group, an interventional system for guiding various minimally invasive procedures based on a clinical US imaging system was developed. Its performance was evaluated using phantoms, and ex vivo tissue samples including human blood cells, porcine fat, nerves and tendons. ${ }^{14-17}$ In this paper, for the first time, an interventional multispectral PA imaging system was used to guide nerve blocks in a swine model in vivo, in which excitation light with two wavelength ranges $(750-900 \mathrm{~nm}$ and $1150-1300 \mathrm{~nm})$ were used to identify nerves and blood vessels.

\section{MATERIALS AND METHODS}

\subsection{The imaging system}

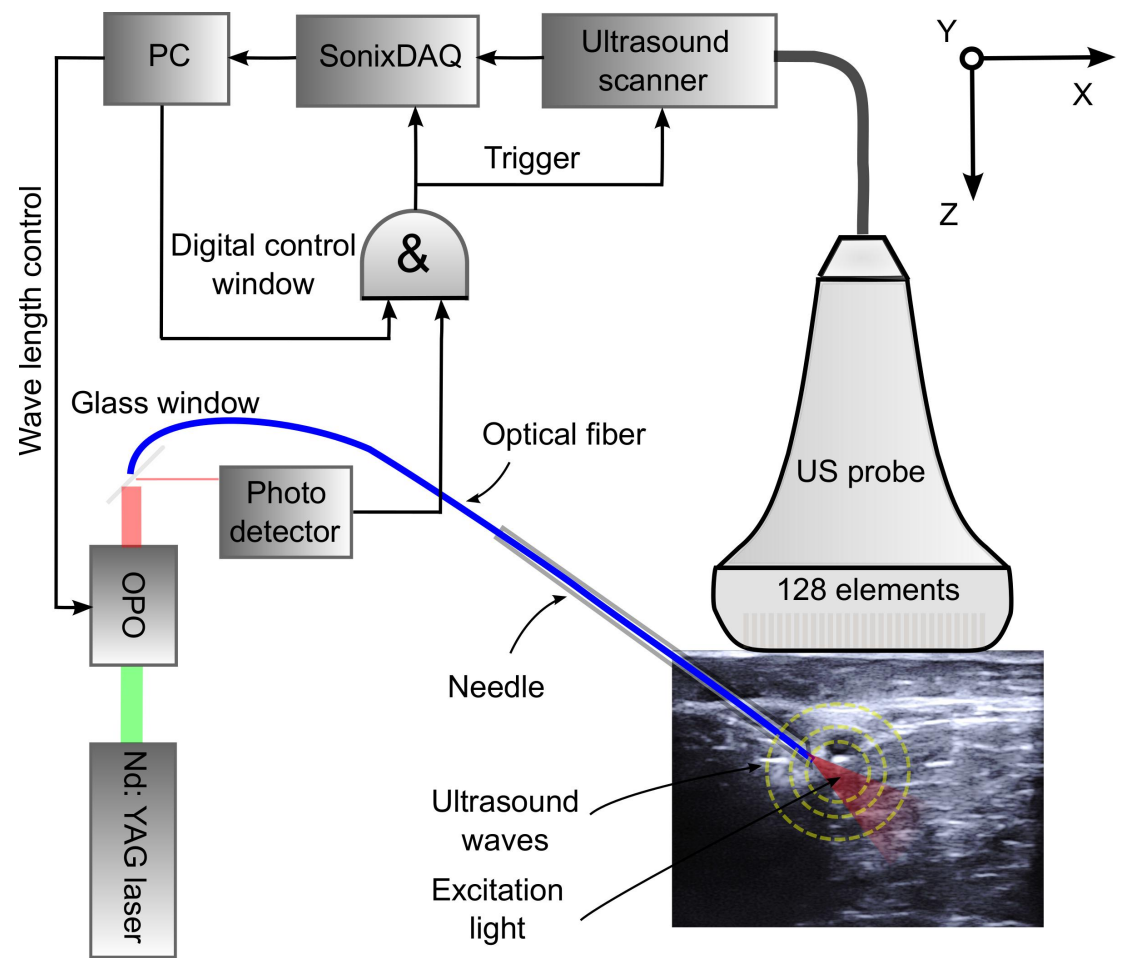

Figure 1: Schematic illustration of the interventional multispectral photoacoustic imaging system.

The imaging system was reported in detail elsewhere; ${ }^{14-16}$ here it is described briefly. The excitation light was provided by an optical parametric oscillator (OPO) system (VersaScan L-532, Spectra-Physics, Santa Clara, CA) pumped by a Nd:YAG laser (pulse width 6 ns, repetition rate $10 \mathrm{~Hz}$, Quanta-Ray, INDI-40-10, SpectraPhysics, Santa Clara, CA). The wavelength-tunable OPO had two output wavelength ranges: the signal (700 - $900 \mathrm{~nm}$ ) and the idler $(1100-2200 \mathrm{~nm})$, with wavelength tuning controlled by a custom Labview program (National Instruments, Berkshire, UK) via a motorized crystal. The OPO outputs were focused onto separate optical fibers, which delivered light to a single fiber that was positioned within the cannula of a 14 gauge spinal needle to deliver light in front of the needle tip (Figure 1).

US detection was based on a commercial US imaging system (SonixMDP, Analogic Ultrasound, Richmond, BC, Canada). To access its low level libraries, the system was operated in its research mode. A 14-5 MHz linear array transducer (L14-5/38, Analogic Ultrasound, Richmond, BC, Canada) was used as the US receiver. Raw data from the transducer was sampled at $40 \mathrm{MHz}$ with a 12-bit ADC by a 128-channel data acquisition system (SonixDAQ, Analogic Ultrasound, Richmond, BC, Canada). Acquisitions of PA and US data were triggered by optical pulses. A digital control window in combination with a logical AND gate was used to control the triggering 
of the US system, to control the speed of imaging. Real-time PA image reconstruction was performed using a custom delay-and-sum beam-forming algorithm. The B-mode US images were reconstructed using electronic beam-forming, and were displayed alongside the PA images. Offline PA image reconstruction was performed using a Fourier-domain reconstruction algorithm implemented within the k-Wave MATLAB toolbox. ${ }^{18}$

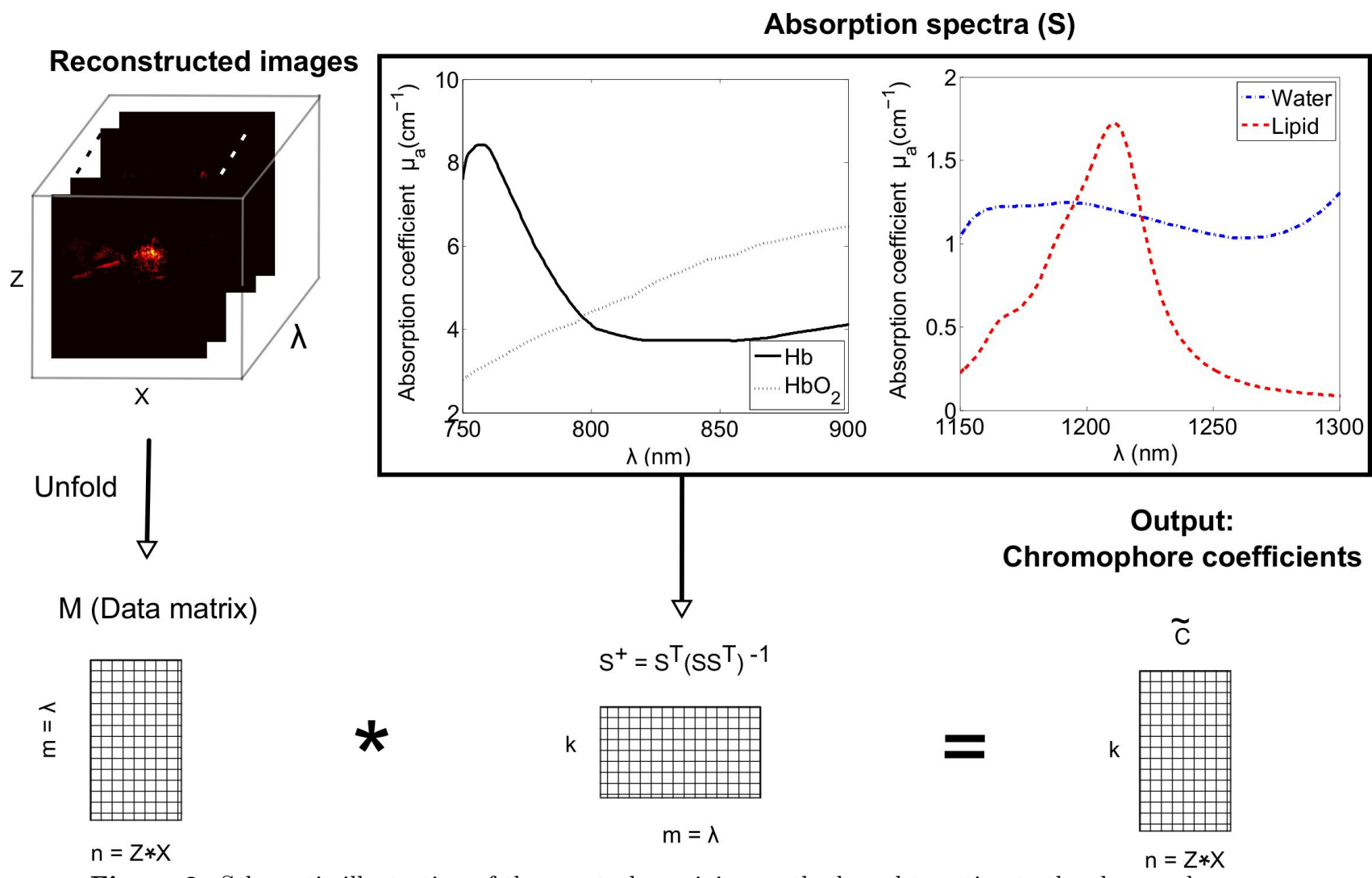

Figure 2: Schematic illustration of the spectral unmixing method used to estimate the chromophore concentration coefficients $\left(\mathrm{HbO}_{2}, \mathrm{Hb}\right.$, water and lipid) from the acquired multispectral photoacoustic imaging data. Spectra data were derived from Ref. ${ }^{20}$

\subsection{Spectral unmixing}

After PA image reconstruction, a multispectral image matrix $M_{n \times m}$ was acquired, in which $n$ is the number of pixels in each PA image, and $m$ is number of excitation light wavelengths. Using a matrix of previously measured optical absorption spectra of chromophores, $S_{k \times m}$, in which $k$ is the number of chromophores considered, and with the assumption of linearity, ${ }^{19}$ we have:

$$
M_{n \times m}=C_{n \times k} * S_{k \times m}
$$

where $C_{n \times k}$ is a matrix of concentration coefficients. An estimate of this matrix, $\tilde{C}_{n \times k}$, is obtained with:

$$
\tilde{C}_{n \times k}=M_{n \times m} * S_{m \times k}^{+}
$$

In which $S_{m \times k}^{+}$is the Moore-Penrose inverse matrix of $S_{k \times m}$ :

$$
S_{m \times k}^{+}=S_{m \times k}^{T}\left(S_{k \times m} S_{m \times k}^{T}\right)^{-1}
$$


In this study, oxygenated and deoxygenated blood were considered as the main contributing chromophores in the wavelength range of 750 - $900 \mathrm{~nm}$; lipids, water were considered as those in the wavelength range of 1150 - $1300 \mathrm{~nm}$. Their optical absorption spectra data was derived from Ref, ${ }^{20}$ and are shown in Figure 2.

(a)

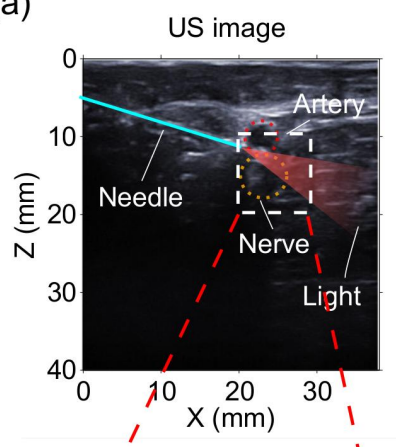

(d)
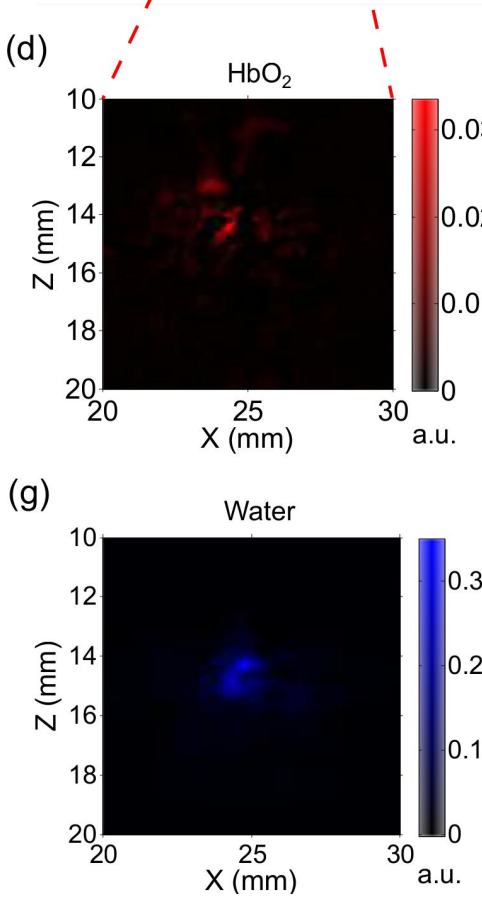

(b)

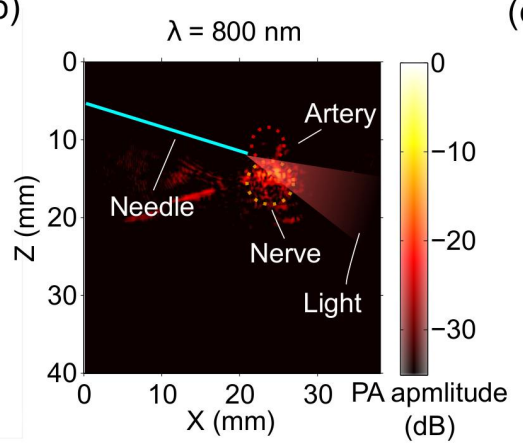

(c)

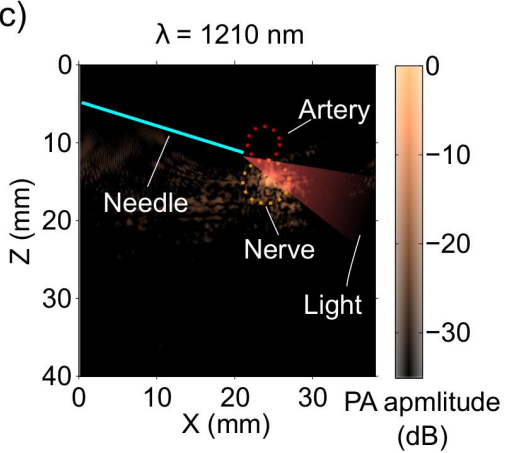

(f)

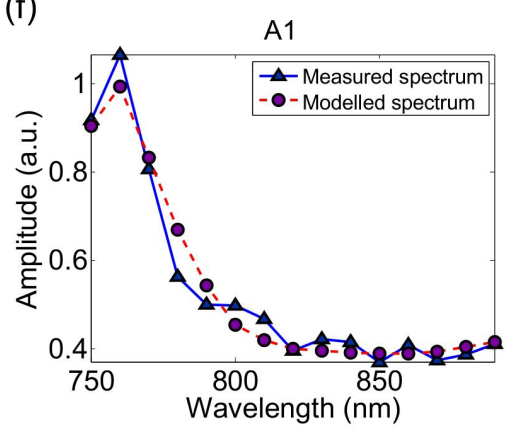

(h)

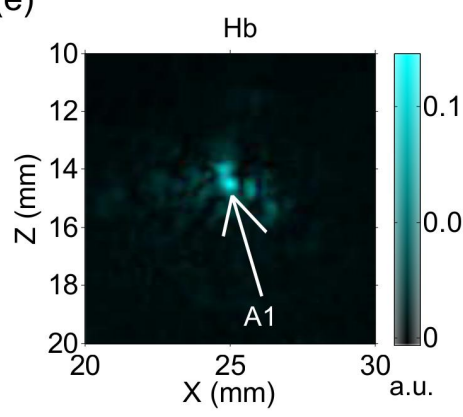

(i)

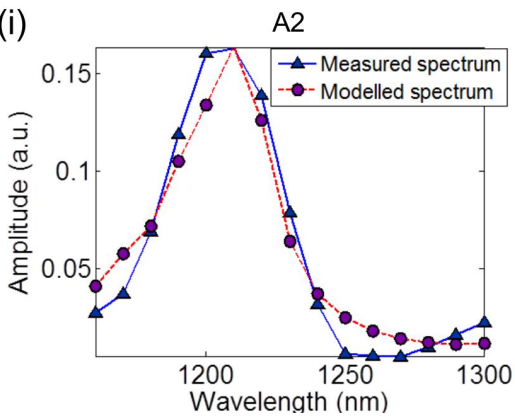

Figure 3: Insertion to the brachial plexus in a swine model in vivo. The ultrasound image (a) and the photoacoustic images acquired with excitation wavelengths of $800 \mathrm{~nm}$ (b) and $1210 \mathrm{~nm}$ (c) were obtained with the same linear-array imaging probe at the surface. Spectral unmixing of the acquired photoacoustic images acquired with excitation wavelengths in the range of 750 - $900 \mathrm{~nm}$ yielded the concentration coefficients of $\mathrm{HbO}_{2}$ (d) and $\mathrm{Hb}(\mathrm{e})$. The measured and modelled spectra at position $\mathrm{A} 1$ (e) were in good agreement (f). Spectral unmixing of the acquired photoacoustic images acquired with excitation wavelengths in the range of $1150-1300 \mathrm{~nm}$ yielded the concentration coefficients of water $(\mathrm{g})$ and lipids $(\mathrm{h})$. The measured and modelled spectra at position A2 (h) were also in good agreement (i), with peaks at $1210 \mathrm{~nm}$.

\subsection{In vivo experiments}

The in vivo swine experiment was performed with ethics approval at the Northwick Park Institute for Medical Research (London, UK). It was performed in a swine model with needle insertions to the brachial plexus under US image guidance. Prior to PA imaging, the close proximity of a nerve to the needle tip was confirmed by nerve stimulation. The wavelength ranges of excitation light were selected based on the optical absorption spectra of lipids, water, oxygenated and deoxygenated blood (Figure 2). PA images with excitation light wavelengths from $750-900 \mathrm{~nm}$ and from $1150-1300 \mathrm{~nm}$ were acquired with wavelength intervals of $10 \mathrm{~nm}$. All experimental 
procedures were conducted according to the UK Home Office regulations and the Guidance for the Operation of Animals (Scientific Procedures) Act (1986).

\section{RESULTS}

With the needle tip positioned in the vicinity of the brachial plexus, an artery was clearly visible in the US image (Figure 3a). A nerve beneath the artery was identified with nerve stimulation, but it was not apparent in the US image. Excitation wavelengths of $800 \mathrm{~nm}$ and $1210 \mathrm{~nm}$ both yielded PA signals from tissue close to the needle tip (Figure 3b,c). Spectral unmixing of multispectral PA images from the wavelength range of 750 - 900 $\mathrm{nm}$ revealed that these PA signals originated primarily from deoxygenated blood (Figure 3d,e), which may have originated from bleeding adjacent to the needle tip. Good agreement between the PA spectra with high signal amplitudes and the linear model [Equation (1)] was observed (Figure 3f). Spectral unmixing of multispectral PA images from the wavelength range of 1150 to $1300 \mathrm{~nm}$ revealed differences in the spatial distributions of water (Figure 3g) and lipids (Figure 3h), with the latter extending farther from the needle tip into the region identified as a nerve. The PA spectra with high signal amplitudes had prominent peaks at $1210 \mathrm{~nm}$, in agreement with modelled data (Figure 3i).

\section{DISCUSSION AND CONCLUSIONS}

We presented, for the first time, interventional multispectral PA imaging of a peripheral nerves in the vicinity of the brachial plexus of a swine model in vivo. Excitation light with wavelength ranges of $750-900 \mathrm{~nm}$ and 1150 - $1300 \mathrm{~nm}$ were used. Spectral unmixing using linear inversion was used to obtain the concentration coefficients for $\mathrm{HbO}_{2}, \mathrm{Hb}$, water and lipids in a region in front of the needle tip. A region with high lipid concentration coefficients was coincident with a neural structure.

The results of this study indicate that lipids are a source of endogenous PA contrast that could be valuable for identifying nerves. However, since lipids are known to also be present in anatomical regions distinct from nerves, such as perineural adipose tissue and fascial planes, anatomical information provided by US imaging could be beneficial. Excitation light delivered through the needle could be beneficial in terms of yielding stronger PA signals in the vicinity of the needle tip, as compared with surface illumination. However, this paradigm has limitations: fluences near the needle tip are high, ${ }^{14}$ and excitation light is provided over a limited spatial region. Delivery of excitation light may prove to be most beneficial for tissue targets that are beyond the imaging depth of PA imaging systems with surface illumination, in conjunction with conventional US imaging. Decreasing the fluence, which could be accomplished using diffusers, will be important for clinical translation. Knowledge of

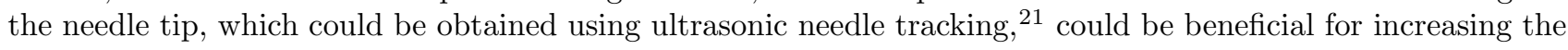
accuracy of spectral unmixing. To assist with the guidance of nerve blocks, multispectral PA imaging could be valuable to supplement US imaging by providing spectroscopic optical specificity.

\section{ACKNOWLEDGMENTS}

This work was supported by an Innovative Engineering for Health award by the Wellcome Trust [WT101957] and the Engineering and Physical Sciences Research Council (EPSRC) [NS/A000027/1], by a Starting Grant from the European Research Council [ERC-2012-StG, Proposal 310970 MOPHIM], and by an EPSRC First Grant [EP/J010952/1].

\section{REFERENCES}

1. L. Helen, et al., "Nerve localization techniques for peripheral nerve block and possible future directions," Acta Anaesthesiol. Scand., 1-13 (2015).

2. D. A. Jamadar, et al., "Musculoskeletal sonography: important imaging pitfalls," AJR 194(1), 216-225 (2009).

3. C. L. Jeng, T. M. Torrillo, and M. A. Rosenblatt, "Complications of peripheral nerve blocks," Br. J. Anaesth. 105(suppl 1), i97-i107 (2010).

4. M. Brynolf, et al., "Optical detection of the brachial plexus for peripheral nerve blocks," Reg. Anesth. Pain Med. 36(4), 350-357 (2011). 
5. A. E. Desjardins, et al., "Needle stylet with integrated optical fibers for spectroscopic contrast during peripheral nerve blocks," J. Biomed. Opt. 16(7), 077004 (2011).

6. A. Balthasar et al., "Optical detection of peripheral nerves: an in vivo human study," Reg. Anesth. Pain Med. 37(3), 277-282 (2012).

7. Y. Wu, et al., "Confocal imaging reveals three-dimensional fine structure difference between ventral and dorsal nerve roots," J. Biomed. Opt. 16(5), 050502 (2011).

8. L. Gao, et al., "Label-free high-resolution imaging of prostate glands and cavernous nerves using coherent anti-Stokes Raman scattering microscopy," Biomed. Opt. Express 2(4), 915-926 (2011).

9. D. T. Raphael, et al., "Images of spinal nerves and adjacent structures with optical coherence tomography: preliminary animal studies," J. Pain 8(10), 767-773 (2007).

10. M. A. Whitney, et al., "Fluorescent peptides highlight peripheral nerves during surgery in mice," Nat. Biotechnol. 29(4), 352-356 (2010).

11. P. C. Beard,"Biomedical photocoustic imaging," Interface Focus 1(4), 602-631 (2011).

12. W. Xia, et al., "Design and evaluation of a laboratory prototype system for 3D photoacoustic full breast tomography," Biomed. Opt. Express 4(11), 2555-2569 (2013).

13. D. Piras, C. Grijsen, P. Schütte, W. Steenbergen, and S. Manohar, "Photoacoustic needle: minimally invasive guidance to biopsy," J. Biomed. Opt. 18(7), 070502 (2013).

14. W. Xia, et al., "Performance characteristics of an interventional multispectral photoacoustic imaging system for guiding minimally invasive procedures," J. Biomed. Opt. 20(8) 086005, (2015).

15. W. Xia, et al., "An interventional multispectral photoacoustic imaging platform for the guidance of minimally invasive procedures," Proc. SPIE 9539 95390D, (2015).

16. W. Xia, et al., "Interventional photoacoustic imaging of the human placenta with ultrasonic tracking for minimally invasive fetal surgeries," Medical Image Computing and Computer-Assisted Intervention-MICCAI 2015, Munich, Germany, (2015)

17. J.-M. Mari, et al., "Interventional multispectral photoacoustic imaging with a clinical ultrasound probe for discriminating nerves and tendons: an ex vivo pilot study," J. Biomed. Opt. 20(11) 110503, (2015).

18. B. E. Treeby, and B. T. Cox, "k-Wave: MATLAB toolbox for the simulation and reconstruction of photoacoustic wave fields," J. Biomed. Opt. 15(2), 021314 (2010).

19. S. Tzoumas, et al., "Unmixing molecular agents from absorbing tissue in multispectral optoacoustic tomography," IEEE Trans. Med. Imag. 33(1) 48-60, (2014)

20. R. Nachabé, et al., "Estimation of lipid and water concentrations in scattering media with diffuse optical spectroscopy from 900 to $1600 \mathrm{~nm}, "$ J. Biomed. Opt. 15(3) 037015, (2010)

21. W. Xia, et al., "In-plane ultrasonic needle tracking using a fiber-optic hydrophone," Med. Phys. 42(10) 5983-5991, (2015) 